\title{
Low-Loss Ultra-Wideband (UWB) Filters Using Suspended Stripline
}

\author{
Wolfgang Menzel ${ }^{1}$, Mohammad S. Rahman Tito ${ }^{1}$, Lei Zhu ${ }^{2}$ \\ ${ }^{1}$ Microwave Techniques, University of Ulm, PO Box, D-89069 Ulm, Germany, wolfgang.menzel@ieee.org \\ ${ }^{2}$ School of Electrical and Electronic Engineering, Nanyang Technological University, Singapore 639798, ezhul@ntu.edu.sg
}

\begin{abstract}
Two types of suspended stripline ultra-wideband bandpass filters are described, one based on a standard lumped element (L-C) filter concept including transmission zeroes to improve the upper passband slope, and a second one consisting of the combination of a low-pass and a high-pass filter.
\end{abstract}

\section{INTRODUCTION}

With the availability of the respective frequency allocations, ultra-wideband (UWB) systems have gained increasing interest for communication and sensor applications. In the frequency range from $3.1 \mathrm{GHz}$ to $10.6 \mathrm{GHz}$, the relative bandwidth of $130 \%$ poses a great challenge for the design of all components. Among these, the realization of band-pass filters (to reduce the spectrum to the allowed band) provides a special problem as with such a bandwidth, transmission line circuits already suffer from higher order resonances. A number of first band-pass filter designs (mostly based on microstrip) have been presented recently [1-5]; these however, occupy a reasonably large substrate area, suffer from high losses, and/or are difficult to manufacture due to very small strips or slots.

On the other hand, suspended stripline (SSL) has proven as a very versatile and low-loss medium for filter design. Not only transmission line resonator filters are available, but also quasilumped filters $[6,7]$ which combine low loss with small circuit size. The basic cross section of SSL as used in this work is shown in Fig. 1. If a SSL component is used as a single component, the substrate may be clamped into a split-block mount as shown in Fig. 1 (a). The overall substrate width is $6 \mathrm{~mm}$, while the mount width is $5 \mathrm{~mm}$. At input and output, a SMA connector is soldered to the SSL. The simulation of such SSL circuits, with good accuracy, can be done with a $2.5 \mathrm{D}$ simulator (e.g.[8]) neglecting the clamping area.

If a SSL component has to be integrated into an extended microstrip circuit, a modified configurations as shown in Fig. 1 (b) can be employed. The SSL component is designed with microstrip ports, maintaining, however, SSL for all filter elements. A small channel according to the length of the SSL portion of the circuit is machined into the carrier plate of the circuit, and a small cap is placed on top of the SSL circuit, together forming the local channel. The conductive connection between metal carrier and cap is achieved by a number of vias fabricated in the substrate.

Making use of both sides of the substrate, different quasilumped circuit elements can be realized [7]. A wide line itself already forms a capacitor to ground, its value can be increased by a ground metallization on the opposite substrate side. Series capacitors are formed by end coupling or broadside coupling. A thin strip acts as an inductor; its value can be increased using an inset into the connecting structure. Typically, the equivalent circuit element values of the lumped elements are extracted by comparing the simulated response of the real SSL structure to that of the respective circuit element.

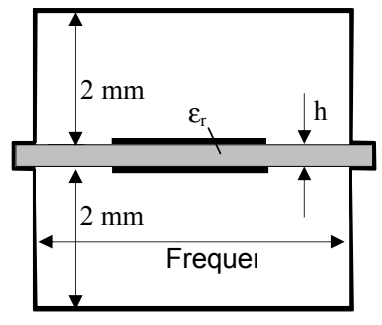

(a)

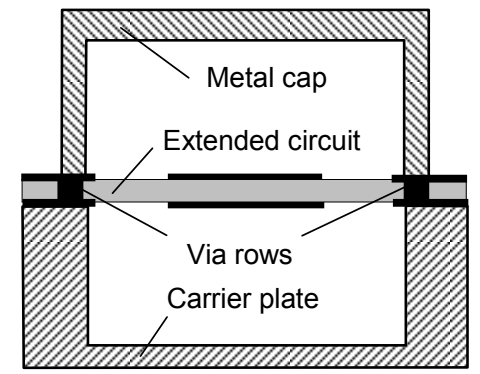

(b)
Fig. 1: Cross section of suspended stripline. (a) test arrangement, (b) possible configuration for implementation into a larger circuit.

\section{FILTER DESIGN AND RESULTS}

\section{A. Standard filter}

A first UWB SSL filter design is based on a standard type filter configuration using series and parallel resonators as indicated in Fig. 2. In addition to the normal filter configuration, two capacitances are added parallel to the inductances of the series resonators to improve the upper passband slope [7]. Fig. 3 shows the two sides of the layout of this filter. The inductances and the capacitances of the series resonators are formed by narrow strips and broadside coupling, respectively. The additional capacitances parallel to the inductances (Fig. 3, connected with dotted lines) are realized by end coupling between input/output lines and the patches for the capacitances. The parallel resonators are formed by relatively large patches with small narrow inductive strips to the side of the mount (the calculation of such resonators is demonstrated in [6]). The original design of this filter can be done using standard filter design, e.g. [9], followed by optimization with a full-wave simulator [8]. For an UWB filter 
as intended here, the design requires small values of the inductances, but quite large capacitances. On the one hand, the small inductances can easily be realized with this circuit concept, on the other hand, the value of the capacitances can be increased with a substrate of a higher dielectric constant. Therefore, a Duroid 6010 substrate with $\varepsilon_{\mathrm{r}}=10.8$ and a thickness of $h=0.254 \mathrm{~mm}$ was selected. The final layout of the filter is given in Fig. 3. The filter alone is only $10.7 \mathrm{~mm}$ long.

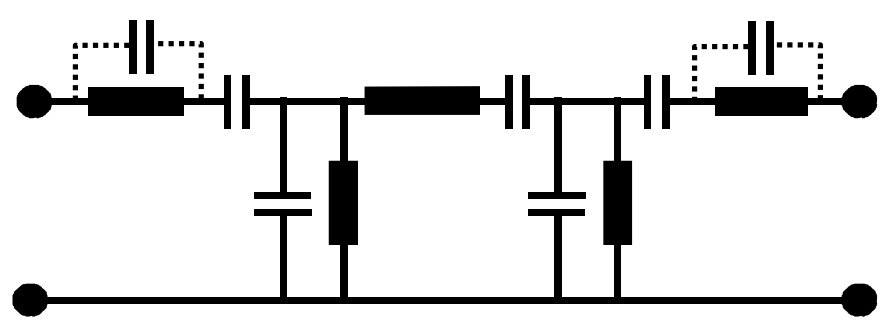

Fig. 2: Equivalent circuit of a five resonator band-pass filter with additional capacitances to achieve transmission zeroes.
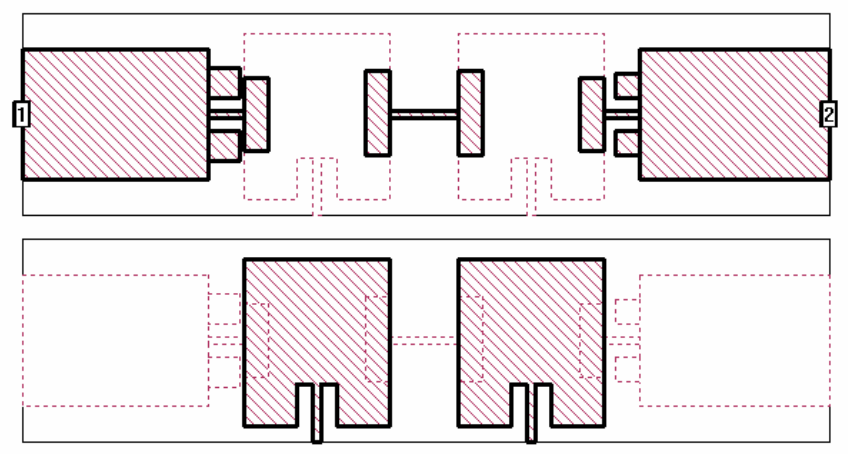

Fig. 3: Top and bottom layout of the filter according to Fig. 2.

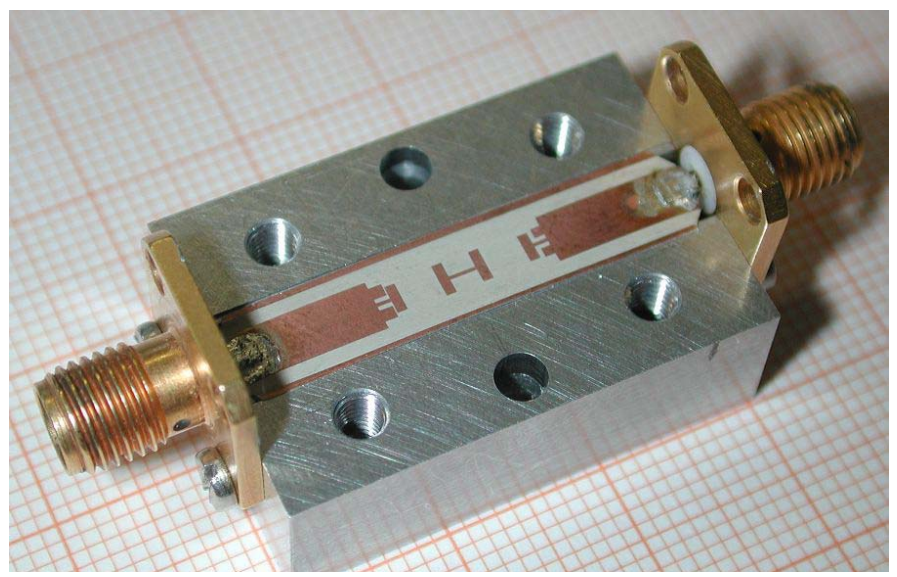

Fig. 4: Photograph of the suspended stripline band-pass filter.
For testing, the filter is placed into a mount of $30 \mathrm{~mm}$ length. A network analyzer calibration was made based on coaxial ports; thus the experimental results include some excess length of transmission line and two transitions from coaxial line to SSL. Fig. 4 shows a photograph of this filter with the mount opened. Simulated and measured results are plotted in Fig. 5. The return loss results slightly higher than intended $(10 \mathrm{~dB})$. Insertion loss is between $0.3 \mathrm{~dB}$ and $0.8 \mathrm{~dB}$, where $0.5 \mathrm{~dB}$ are caused by the return loss. A considerable improvement of the upper passband slope has been achieved due to two transmission zeroes around $13 \mathrm{GHz}$. Although this filter is based on a quasi-lumped approach, spurious responses appear at frequencies above $14 \mathrm{GHz}$. Depending on the overall system, these responses may be suppressed by the performance of other components like amplifiers, or an additional low-pass filter may be added (as it will be shown as part of the next filter design).

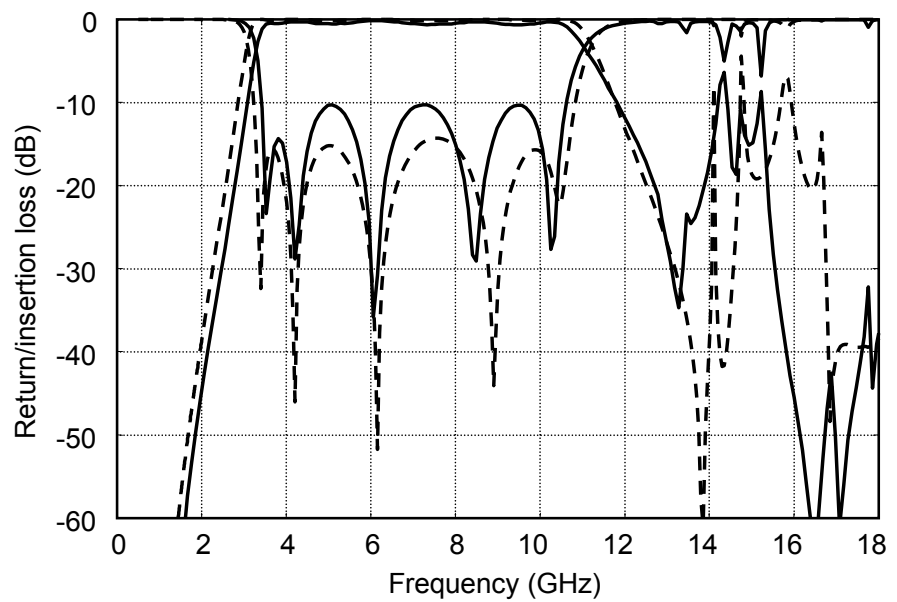

Fig. 5: Simulated (dashed lines) and measured (solid lines) performance of the suspended stripline band-pass filter.

\section{B. Combination of low-pass and high-pass filters}

A second possibility for the design of UWB filters is the combination of a low-pass and a high-pass filter as presented in [4] in microstrip technology. Once again, very compact and low-loss filters of this type can be realized in SSL. For the lowpass filter section, very high characteristic impedances can be achieved with narrow strip, while very low impedances are possible with wide strips backed by a ground conductor on the opposite substrate side. This results in small circuit size and a wide stopband. Once again, filter design starts with a standard L-C equivalent circuit, from which the practical geometry is derived including the discontinuity effects. An optimization based on a full-wave simulator results in the final circuit.

For the high-pass filter section, strong broadside coupling between wide line sections on opposite sides of the substrate are employed together with thin strips to ground, combined with an inset to increase the available inductance. 

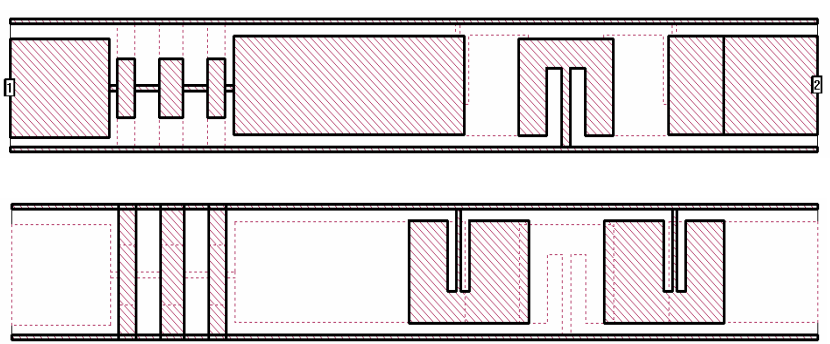

Fig. 6: Top and bottom layout of the band-pass filter composed of low-pass (left side) and high-pass (right side) filter.

The layout of the resulting filter structure for both sides of the substrates is given in Fig. 6. The low-pass section (left side of the structure) has seven elements and is only $5.05 \mathrm{~mm}$ long. The high-pass filter part (right side of the structure) comprises four capacitances based on broadside coupling and three inductances with deep insets to achieve the necessary values for the inductances. Total high-pass filter length amounts to $12.75 \mathrm{~mm}$. To improve the resulting return loss when combining the two filters, a section of transmission line of $7 \mathrm{~mm}$ length was added between the two filter parts, resulting in an overall filter length of $24.8 \mathrm{~mm}$.

As substrate material, RO 4003 with a substrate thickness of $\mathrm{h}=0.203 \mathrm{~mm}$ and a dielectric constant of $\varepsilon_{\mathrm{r}}=3.38$ was selected. (This substrate is fully compatible with standard PCB fabrication processes including vias; thus a configuration according to Fig. 1 (b) is possible). The performance of both low-pass and high-pass filter simulated separately is plotted in Fig. 7. Both filters have been designed for a return loss better than $20 \mathrm{~dB}$.

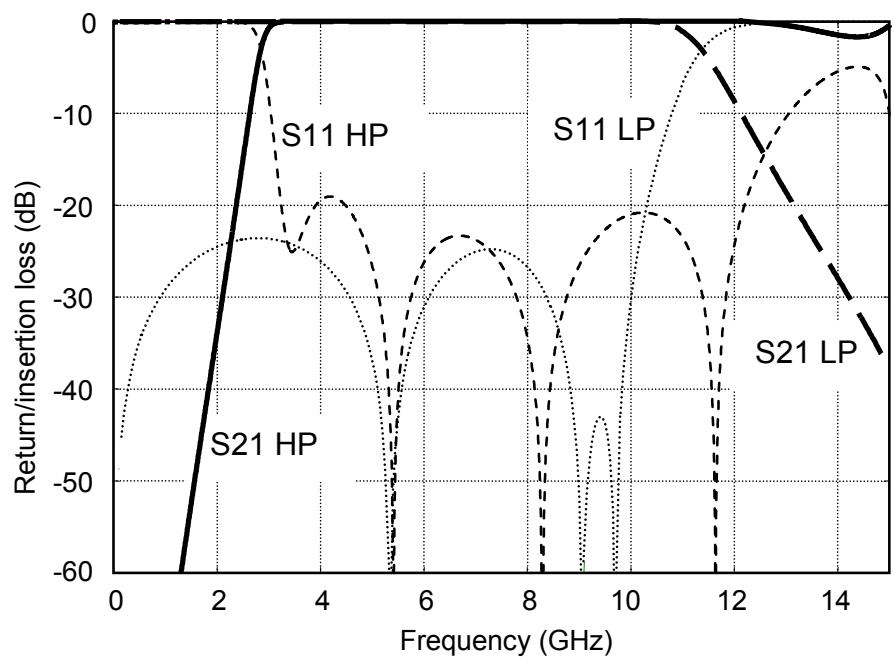

Fig. 7: Simulated performance of the suspended stripline low-pass (LP) and high-pass (HP) filters.

A photograph of the combined low-pass/high-pass filter is presented in Fig. 8. The elements of the low-pass section clearly are visible on the left filter side; from the high-pass section, only part of the structure is visible on the top side.

A simulation of the complete filter was done as well to account for the interaction between the two different filter sections. Both the complete simulation results as well as the measured filter performance are plotted in Fig. 9. An excellent agreement can be stated between theory and experiment. The hump between $14 \mathrm{GHz}$ and $15 \mathrm{GHz}$ is due to the interaction of the two filter sections. In the investigated frequency range up to $20 \mathrm{GHz}$, isolation is better than $40 \mathrm{~dB}$. Insertion loss is lower than $0.4 \mathrm{~dB}$ in the complete passband.

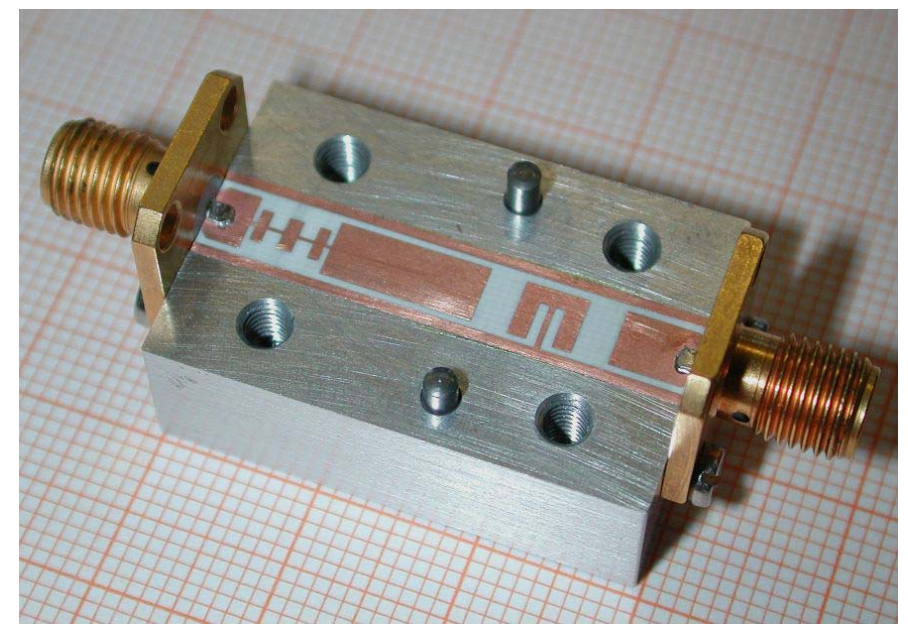

Fig. 8: Photograph of the suspended stripline band-pass filter consisting of low-pass (left side) and high-pass (right side) filter.

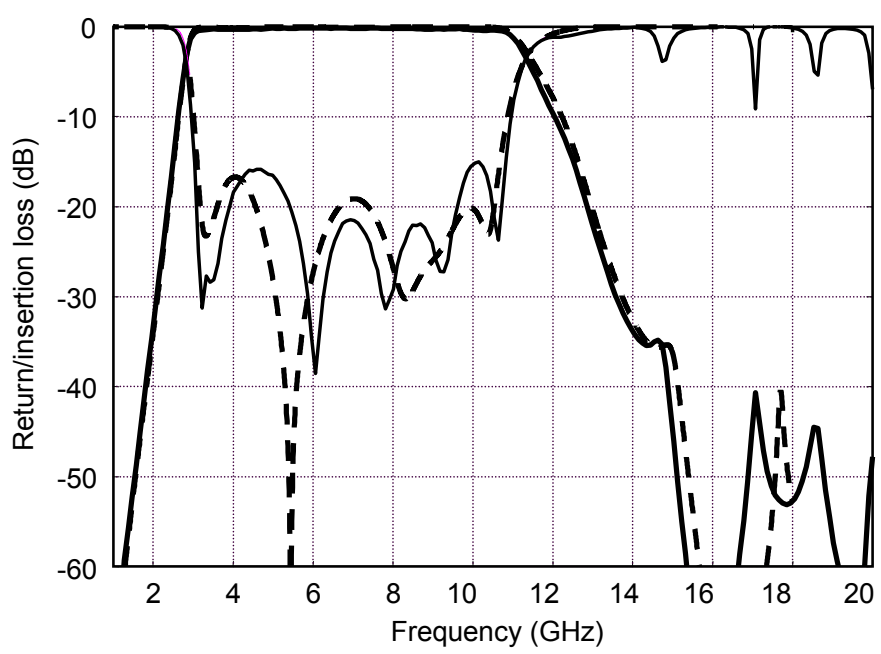

Fig. 9: Simulated (dashed lines) and measured (solid lines) performance of the suspended stripline band-pass filter consisting of low-pass and high-pass filter.

Group delay variation of this filter is below $0.14 \mathrm{~ns}$ in the frequency range between $4 \mathrm{GHz}$ and $10.6 \mathrm{GHz}$, it increases below $4 \mathrm{GHz}$ due to the steep slope of the high-pass filter with an order of seven. If necessary, this can be improved by replacing this high-pass filter by one with a lower order, e.g. of 
order three. The layout of such a modified band-pass filter is given in Fig. 10. Due to the smaller high-pass filter and a shorter distance between the two filter parts, this filter has a length of only $13 \mathrm{~mm}$. Simulated and measured performance is given in Fig. 11, and the simulated group delay is plotted in Fig 12 (measured group delay, except for some ripple and some shift due to longer connecting lines, is very similar to these results). In the complete passband, group delay variation is smaller than $0.14 \mathrm{~ns}$.

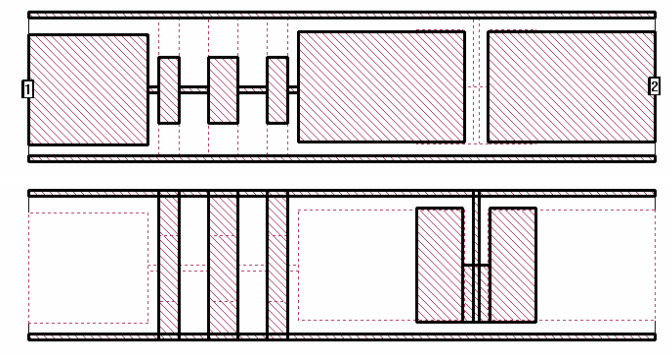

Fig. 10: Top and bottom layout of the modified band-pass filter composed of low-pass (left side) and high-pass (right side) filter.

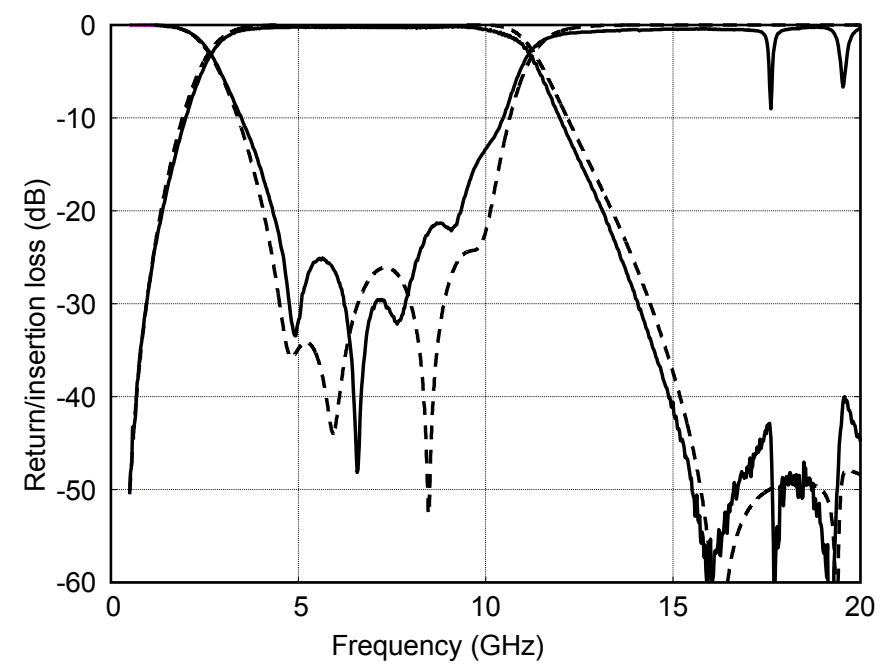

Fig. 11: Simulated (dashed lines) and measured (solid lines) performance of the suspended stripline band-pass filter according to Fig. 10.

\section{CONCLUSION}

Design, realization, and experimental results of two suspended stripline ultra-wideband band-pass filters have been presented. One filters is based on a straightforward L-C lumped-element design, including additional capacitive coupling to improve the upper passband slope. The second type of filter is a combination of a low-pass and a high-pass filter. The first two filters result in a very compact setup of $12.7 \mathrm{~mm}$ and $24.8 \mathrm{~mm}$ length, respectively. Insertion loss is very low; the contributions of conductor and dielectric loss (including excessive lengths of transmission line and the transitions to coaxial waveguide) amount to $0.3 \ldots 0.4 \mathrm{~dB}$ only; in the case of the first filter, $0.5 \mathrm{~dB}$ partly are added due to a return loss of
$10 \mathrm{~dB}$ only. Finally, results are given of a modified filter (lowpass/highpass combination) with improved group delay variation.

The filters as presented here are discrete components in a specific mount, but it has been indicated how such filters can be integrated into larger microstrip circuits. The same principles can be transferred to an LTCC circuit with at least three layers, at the expense, however, of higher losses.

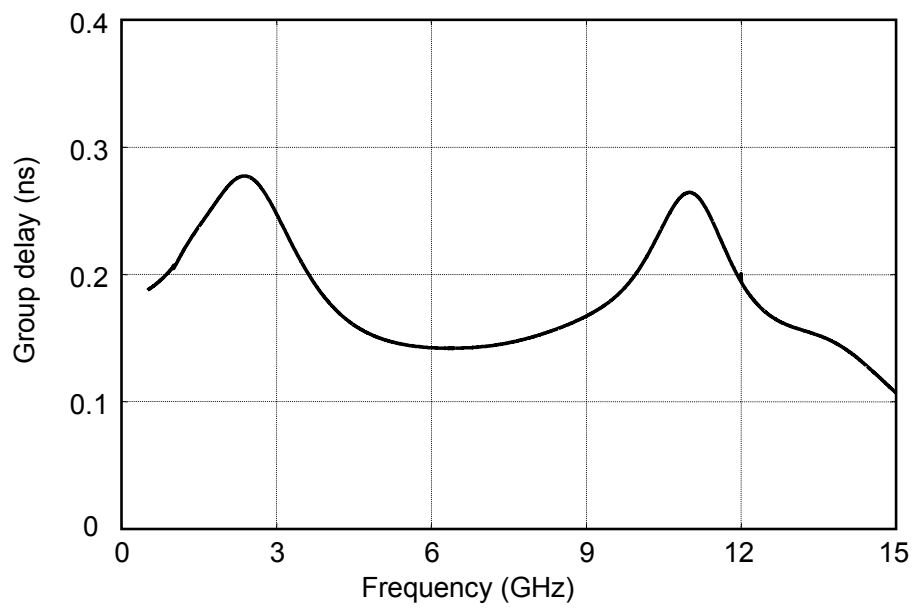

Fig. 12: Simulated group delay of the new filter according to Fig. 10.

\section{REFERENCES}

[1] A. Saito, H. Harada and A. Nishikata, "Development of band pass filter for ultra wideband (UWB) communication," in IEEE Conf. on Ultra Wideband Systems and Tech., 2003, pp. 76-80.

[2] H. Ishida and K. Araki, "Design and analysis of UWB bandpass filter with ring filter," in 2004 IEEE MTT-S Int. Microwave Symp. Dig., pp.1307-1310, June 2004.

[3] C.-L. Hsu, F.-C. Hsu and J.-T. Kuo, "Microstrip bandpass filters for ultra-wideband (UWB) wireless communications," in 2005 IEEE MTT-S Int. Microwave Symp. Dig., WE2F-2, June 2005.

[4] K. Li, D. Kurita and T. Matsui, "An ultra-wideband bandpass filter using broadside-coupled microstrip-coplanar waveguide structure," in 2005 IEEE MTT-S Int. Microwave Symp. Dig., WE2F-1, June 2005.

[5] L. Zhu, S. Sun and W. Menzel, "Ultra-wideband (UWB) bandpass filters using multiple-mode resonator," accepted for publication in the IEEE Microwave Wireless Components Letters.

[6] W. Menzel, "A Novel Miniature Suspended Stripline Filter," European Microwave Conf., Munich, Oct. 2003, pp. 1047 - 1050.

[7] W. Menzel, A. Balalem, "Quasi-Lumped Suspended Stripline Filters and Diplexers," accepted for publication in the IEEE Trans. on Microw. Theory Tech..

[8] SONNET, Version 10, Sonnet Software Inc.

[9] G. Matthaei, L. Young, E.M.T. Jones, "Microwave filters, impedance matching networks, and coupling structures," Reprint by Artech House, 1980. 